\title{
Critical Nodes Identification in Complex Networks
}

\author{
Haihua Yang ${ }^{1,2}$ and Shi An ${ }^{1, *}$ \\ 1 School of Transportation Science and Engineering, Harbin Institute of Technology, Harbin 150090, China; \\ haihua.yang@connect.polyu.hk or SmileYang1991@163.com \\ 2 Department of Civil and Environmental Engineering, The Hong Kong Polytechnic University, Hung Hom \\ 999077, Hong Kong, China \\ * Correspondence: 16b932003@stu.hit.edu.cn
}

Received: 25 December 2019; Accepted: 6 January 2020; Published: 8 January 2020

\begin{abstract}
Critical nodes identification in complex networks is significance for studying the survivability and robustness of networks. The previous studies on structural hole theory uncovered that structural holes are gaps between a group of indirectly connected nodes and intermediaries that fill the holes and serve as brokers for information exchange. In this paper, we leverage the property of structural hole to design a heuristic algorithm based on local information of the network topology to identify node importance in undirected and unweighted network, whose adjacency matrix is symmetric. In the algorithm, a node with a larger degree and greater number of structural holes associated with it, achieves a higher importance ranking. Six real networks are used as test data. The experimental results show that the proposed method not only has low computational complexity, but also outperforms degree centrality, k-shell method, mapping entropy centrality, the collective influence algorithm, DDN algorithm that based on node degree and their neighbors, and random ranking method in identifying node importance for network connectivity in complex networks.
\end{abstract}

Keywords: network disintegration; network connectivity; node importance; structure hole

\section{Introduction}

Networks have become an attractive subject in complex system research, due to their universality for depicting a wide range of systems in nature and society [1-3]. This is based on the fact that a complex system which is composed of a set of components with connections between them can be graphically represent by a network graph, where nodes represent individual components and links stand for their interactions.

Networks are everywhere [4,5], ranging from nature and biological system to society, such as cellular networks [6], traffic networks [7-9], social networks [10], power grids [11], and many others. Research regarding evaluation of node importance for certain structural or functional objectives in complex network is a challenge of both theoretical and practical significance. In real life, complex networks or systems are not always secure. Threats to complex networks can come from two sources, random failures and target destruction [12,13]. Previous studies show that a large number of real systems such as the World Wide Web, the Internet, power grids, and airline networks, etc. are with notable heterogeneity [14], which means that the role of nodes in network structure and function varies greatly. These networks have a higher tolerance to random failures but are sensitive to intentional attacks.

Critical nodes are those special nodes that can affect the structure and function of the network to a greater extent. Protecting the key nodes of the network is of great significance for improving the robustness and survivability of the network [15]. How to design an effective algorithm to evaluate the importance of nodes has attracted a large number of scholars to study it in recent years [16]. Many methods have been proposed from different aspects to identify critical nodes, such as degree centrality (Deg) [17], 
mapping entropy centrality (ME) [18], the collective influence algorithm (CI) [19], semi-local centrality [20], coreness [21], and H-index [22] which are based on node local neighbors information. Eccentricity [23], information indices [24], Katz centrality [25], closeness centrality, betweenness centrality [26], and subgraph centrality [27] are designed from the perspective of number of paths for communication. In addition to the methods mentioned above, there are also many iterative refinement algorithms, which consider not only the number of neighbors but also the importance of its neighbors. Representative algorithms include the well-known PageRank [28], HITs [29], and eigenvector centrality [30], as well as some recently porposed algorithms such as LeaderRank [31] and TwitterRank [32].

Due to the rapid development of information technology, as well as the constantly expanding network scale and increasing complexity of the network, the design for evaluating algorithms on node importance in very large-scale networks is becoming a significant challenge nowadays. The importance evaluation of algorithms is computationally expensive if they are based on global information of the network. Therefore, the design of evaluation algorithm should not only be effective but also efficient. To address this, designing a sorting algorithm based on network local information would be a better solution. In this paper, we present a new approach called DSHC (based on local degree information and structure hole count) to evaluate node importance of the network. We summarize the following three contributions of the paper:

(1) We draw inspiration from the theory of structural holes [33] and only consider the node's local neighborhood information to evaluate the importance of nodes. This makes the algorithm more computationally attractive for large-scale networks;

(2) The proposed algorithm can effectively identify the hubs with numerous structural holes, which play important role in bridging different clusters of the network;

(3) Empirical analyses on real and synthetic networks demonstrate that the proposed method can outperform Deg, k-shell, ME, CI, DDN [34], and random ranking method (Rand).

The outline of this paper is organized as follows: First, we introduce a theory for the study of competition relationship in social networks analysis according to the theory of structural holes and then, devise the evaluation algorithm to identify node importance and introduce the benchmark algorithms in Section 2. In Section 3, we introduce the indicators used to evaluate the accuracy of importance ranking. In Section 4, we present real network datasets for experiments. In Section 5, we compare the proposed algorithms with other existing methods in both synthetic networks and real networks. Finally, we provide a summary in Section 6.

\section{Materials and Methods}

An unweighted and undirected network $G(V, E)$ with $N=|V|$ nodes and $M=|E|$ edges is considered in this paper. The network could be described by an adjacent matrix $A\left(a_{i j}\right)$, where $a_{i j}=1$ represents there is a connection linking node $i$ and node $j$, and $a_{i j}=0$ otherwise. The degree value of node $i$ is represented by $k_{i}$.

\subsection{Measurement of Node Importance Based on Degree and Structural Hole Count}

We begin our analysis by introducing a theory for the study of competitive relationships in social networks, based on the so-called structural holes. From a sociological perspective, structural holes are gaps between a group of indirectly connected nodes, and some individuals that act as structural hole spanners to fill the hole get more network benefits than their neighbors. Take Figure 1 as an example, there are three structural holes marked by dashed arrows associated with the intermediate node Ego. Compared with its neighbors A, B, C, and D, Ego will gain more network benefits than its neighbors, because there is no alternative communication channel between them. Obviously, the Ego node plays a significant role in maintaining the network connectivity and we can infer that the large degree value a node has and the greater the number of structural holes associated with the node, the more significant the node will be. 


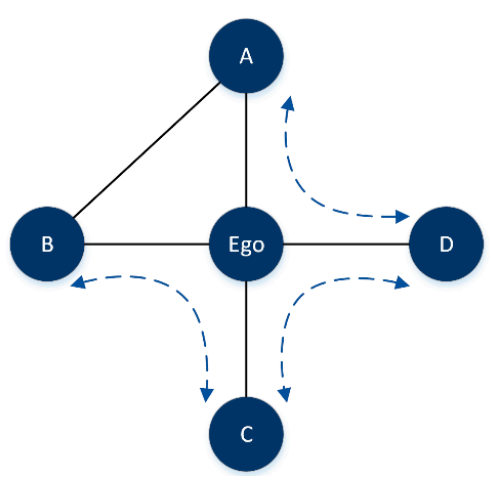

Figure 1. Concept of structural hole.

In view of the analysis above, we leverage the property of structural holes to design an intuitive algorithm for quantifying node importance in maintaining the network connectivity, based on degree and structural hole count, expressed by

$$
\operatorname{DSHC}_{i}=\sum_{j \in \Gamma_{i}}\left(\left(\frac{1}{k_{i}}+\frac{1}{k_{j}}\right) * \frac{1}{1+\Delta_{i j}}\right)^{2}
$$

where $\Gamma_{i}$ represents the neighbor set of node $i$ and $\Delta_{i j}$ represents the number of structural holes formed between node $i$ and $j$ with node $i$ as the intermediary. According to Equation (1), the larger the degree of a node and its neighbors, and the higher the number of structural holes between the node and its neighbors, this means that the stronger the irreplaceability of nodes in the structure, the smaller the value of $D S H C$ will be. According to Figure 1, we calculate the $D S H C_{E g o \_} A$ between node Ego and node A, there are two structural holes between node Ego and node A, $\{$ A-Ego-C, A-Ego-D $\}$. Therefore, we can get

$$
D_{S H C}{ }_{E g o \_} A=((1 / 4+1 / 2) \times(1 /(1+2)))^{2}=1 / 16
$$

Futhermore, we calculate the $D S H C_{E g o_{-} B}, D S H C_{E g o_{-} C}$, and $D S H C_{E g o_{-} D}$, and sum them to get $D_{S H C}$ Ego.

The algorithm comprehensively considers the degree of the node and its neighbors and the information about the topological overlapping of its neighbors. A large DSHC value indicates that the neighborhood of the node is closely interconnected and forms a dense local cluster. Removal of such nodes usually does not significantly affect the network connectivity.

The DSHC algorithm is described as follows:

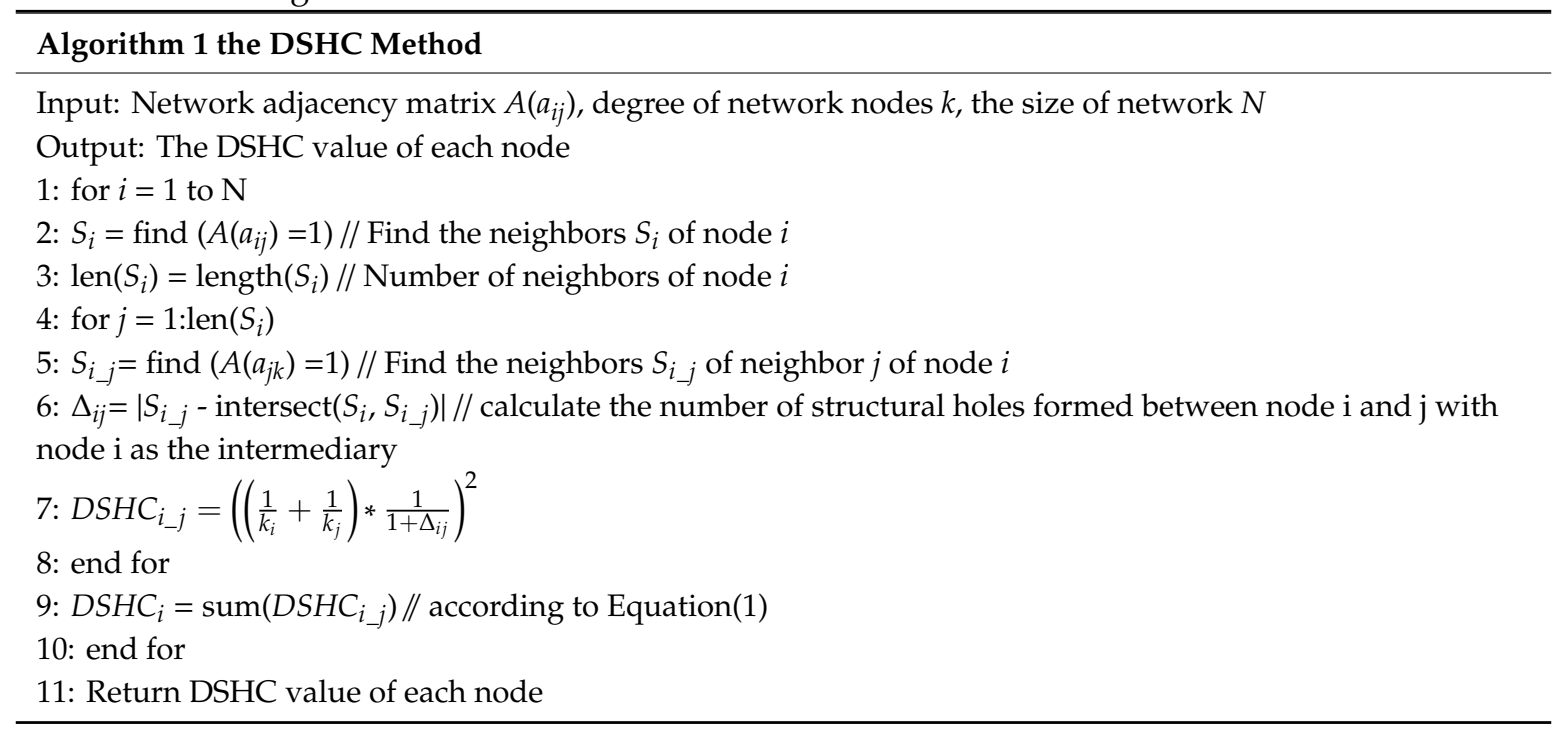




\subsection{Benchmark Methods}

We use several popular heuristic methods that are also based solely on the network topology to investigate the performance of the proposed method. These measures include:

1. Degree centrality

Degree centrality is a basic ranking algorithm to evaluate the importance of nodes. The degree of node $i$ can be defined as

$$
k_{i}=\sum_{j=1}^{N} a_{i j}
$$

2. The k-shell decomposition method

The k-shell decomposition algorithm categorizes the nodes into core nodes and fringe nodes. The algorithm steps are as follows: First, delete all nodes that have only one connection and assign those nodes to the 1-shell. In this process, there may be new nodes with $k=1$, and then pruning is repeated until all nodes with $k=1$ are removed. The removed nodes will be classified using a 1-shell. Next, this process continues in a similar way for nodes with degree $k=2$ and gets the 2-shell of the network. The pruning is repeated until all network nodes are assigned to one of the shells.

3. The DDN method

The DDN method believes that the importance of a node is determined by the degree of the node and the degree of its neighbors, which is defined as

$$
D D N_{i}=\sum_{j \in \Gamma_{i}} w_{i j}
$$

where the weight of node is expressed as $w_{i j}=k_{i} \times k_{j}$.

4. The ME centrality

The ME centrality is based on local neighborhood information, which is defined as

$$
M E_{i}=-k_{i} \sum_{j=1}^{P} \log k_{j}
$$

where $P$ is the number of node $i$ 's neighbors.

\section{The $\mathrm{CI}$ algorithm}

The CI algorithm can be utilized to identify a set of key nodes whose removal is most efficient in destroying the network connectivity, which is defined as

$$
C I_{i}=\left(k_{i}-1\right) \sum_{j \in \partial b a l l(i, \ell)}\left(k_{j}-1\right)
$$

where $\partial b a l l(i, \ell)$ is the boundary of the sphere centered on node $i$ with radius $\ell$, and is composed of all nodes whose distance from node $i$ does not exceed $\ell$. The $C I$ radius of this article is set to 2 .

6. Random ranking method

Ranking the importance of network nodes in a random order. 


\section{How to Evaluate the Performance}

There are three kinds of metrics which are often used to evaluate the performance of ranking algorithm, namely susceptibility value $S$ [35], the maximum connectivity coefficient [36] $G$, and the network efficiency $[37,38] \eta$. The change of network connectivity caused by removing node is equivalent to the importance of node.

1. The maximum connectivity coefficient

The maximum connectivity coefficient reflects the size of the maximum connected component of the network, which is attacked by removing node. Mathematically reads

$$
G=R / N
$$

where $R$ means the size of the giant component after node removal and $N$ represents the number of nodes in the network. Obviously, the smaller $G$ is, the better the attack strategy is.

2. Susceptibility

Susceptibility quantifies the change of network connectivity in response to the removal of network nodes. The gradual removal of network nodes breaks the network into many disconnected parts. In this process, the susceptibility value, $\mathrm{S}$, usually has a peak value corresponding to a specific proportion, pc, at which the network collapse. In particular, if the network is broken multiple times during the process of gradually removing nodes, there exists multiple peaks. The susceptibility value $S$ is defined as:

$$
S=\sum_{s<\sigma} \frac{n_{s} s^{2}}{N}
$$

where $n_{s}$ denotes the number of components with size equal to $s$ and $N$ represents the size of the network nodes. Obviously, the smaller the value of $p_{c}$, the better the ranking algorithm.

3. The Decline Rate of Network Efficiency

Network efficiency reflects the quality of the network connectivity, which is defined as

$$
u=\frac{1}{N(N-1)} \sum_{i, j \in V} \eta_{i j}
$$

where $\eta_{i j}$ means the efficiency of node $i$ and $j, \eta_{i j}=1 / d_{i j}$, and $d_{i j}$ denotes the shortest path length between node $i$ and $j$. As network nodes are gradually removed, the average shortest path between nodes becomes larger, which makes the connectivity of the network worse.

We adopt the decline rate of the network efficiency to analyze the disintegration effect of nodes removal, which is defined as

$$
\eta=1-u / u_{0}
$$

where $\eta$ represents the efficiency of the network after network attack and $u_{0}$ denotes the network efficiency of the original network.

\section{Data Description}

To validate the effectiveness of the DSHC method, six real complex networks including Facebook (Slavo Zitnik's social network on Facebook) [39], Erdos (scientific collaboration network) [40], USAir (American aviation network) [41,42], USAirport (USA airport network) [43], Yeast (yeast protein-protein binding network) [44,45], and Power (connections between US west power stations) [46] are used for a comparison experiment. The basic statistics of six real networks are shown in Table 1. 
Table 1. The basic statistical features of Facebook, Erdos, USAir, USAirport, Yeast, Power networks where $N$ and $M$ represent the number of nodes and connections, respectively. $<k>$ and $C$ is the average degree and average clustering coefficient of the network, $k s_{\text {max }}$ represents the largest $k$-shell values, and $L$ denotes the average shortest path length.

\begin{tabular}{ccccccc}
\hline Network & $\boldsymbol{N}$ & $\boldsymbol{M}$ & $\boldsymbol{k} \boldsymbol{k}$ & $\boldsymbol{C}$ & $\boldsymbol{k} \boldsymbol{s}_{\max }$ & $\boldsymbol{L}$ \\
\hline USAir & 332 & 2126 & 12.807 & 0.625 & 26 & 2.738 \\
Facebook & 324 & 2218 & 13.69 & 0.466 & 18 & 3.054 \\
Erdos & 446 & 1417 & 6.33 & 0.296 & 9 & 3.952 \\
USAirport & 1574 & 28,236 & 21.901 & 0.505 & 64 & 3.113 \\
Yeast & 2375 & 11,693 & 9.847 & 0.306 & 40 & 5.094 \\
Power & 4961 & 6964 & 2.669 & 0.080 & 5 & 18.989 \\
\hline
\end{tabular}

\section{Results and Analysis}

In order to verify the effectiveness of the DSHC indicator in measuring the importance of nodes, we compare the proposed method with K-shell, Degree, ME, CI, and Rand to simulate the intentional attack effect on real and synthetic networks by selectively removing the nodes in descending order of node importance. The experiments were simulated on MATLAB toolset.

\subsection{Experiments in Real Networks}

The experimental results in Figure 2 reflect the performance of different attack strategies on six real networks in terms of the maximum connectivity coefficient, which is estimated by numerical simulation of the change of relative size of the giant component of the network, as mentioned previously. It can be seen that in all six networks, using DSHC indicators to remove important nodes in the network, can make the scale of the network giant change the fastest. This indicates that the proposed DSHC index can identify the most important nodes more accurately in the network than the k-shell, degree, ME, CI, DDN, and Rand. For example, in the Yeast network in Figure 2, when $p=0.2$, the $G$ values of the k-shell, degree, ME, CI, DDN, DSHC, and Rand are 0.7154, 0.5314, 0.5903, 0.5781, 0.6143, 0.2080, and 0.7229 , respectively, which indicates that the network connectivity becomes worst as compared with the k-shell, degree, ME, CI, DDN, and Rand when using the DSHC method to remove the top $20 \%$ nodes. The attack effectiveness of DSHC is 70.92\%, 71.23\% higher than that of k-shell and Rand respectively. Similarly, in power network, when $p=0.1$, the $G$ values of the indicators k-shell, degree, ME, CI, DDN, DSHC, and Rand are $0.7891,0.1358,0.2374,0.3111,0.3036,0.0498$, and 0.8197 , respectively. This shows that when the top $10 \%$ of nodes are removed according to the DSHC index, the network connectivity is also the worst as compared with the top $10 \%$ of nodes removed according to the k-shell, degree, ME, CI, DDN, and Rand algorithms. 

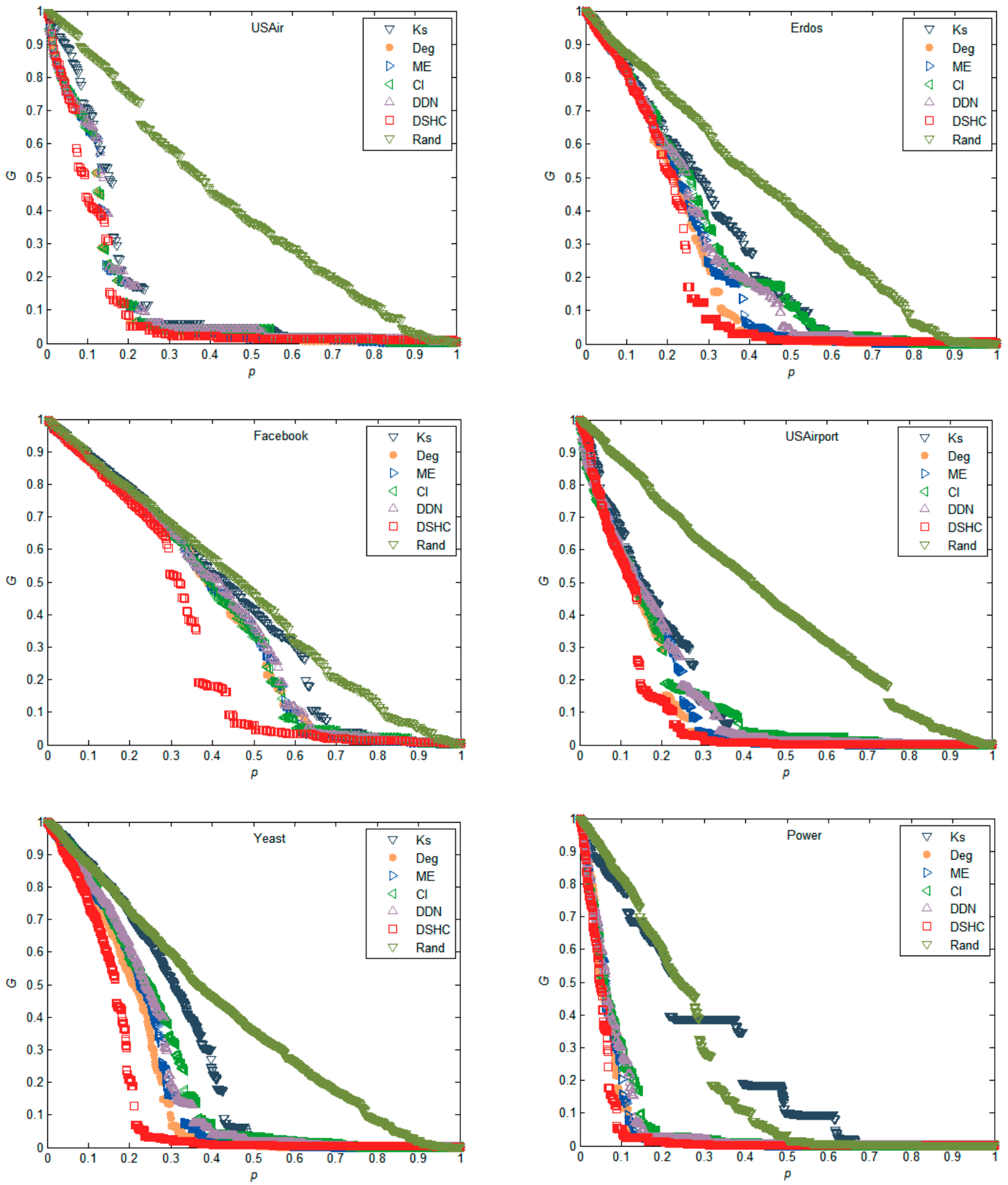

Figure 2. The network maximum connectivity coefficient $(G)$ as a monotonic function of the number of removed nodes as compared with k-shell, degree, mapping entropy centrality (ME), CI, DDN, and Rand in USAir, Erdos, Facebook, USAirport, Yeast, and Power networks, respectively.

Figure 3 shows the susceptibility of the resulting networks as a function of proportion $p$ of nodes removed, using different attack strategies, as discussed above. We can see that the DSHC method outperforms other methods in that the network collapses into many disconnected pieces with the minimum $p$. More specifically, the susceptibility reaches the maximum by removing about $3 \%$ of the nodes with the proposed method in the power network (Figure 3). In contrast, the values, $p$, corresponding to the maximum susceptibility value of the $\mathrm{CI}$ and degree index are $3.56 \%$ and $4.09 \%$, respectively. Overall, the performances of random schemes are inferior; the degree centrality can outperform the $\mathrm{CI}$ index, and our approach always outperforms other competing methods in these real networks. 

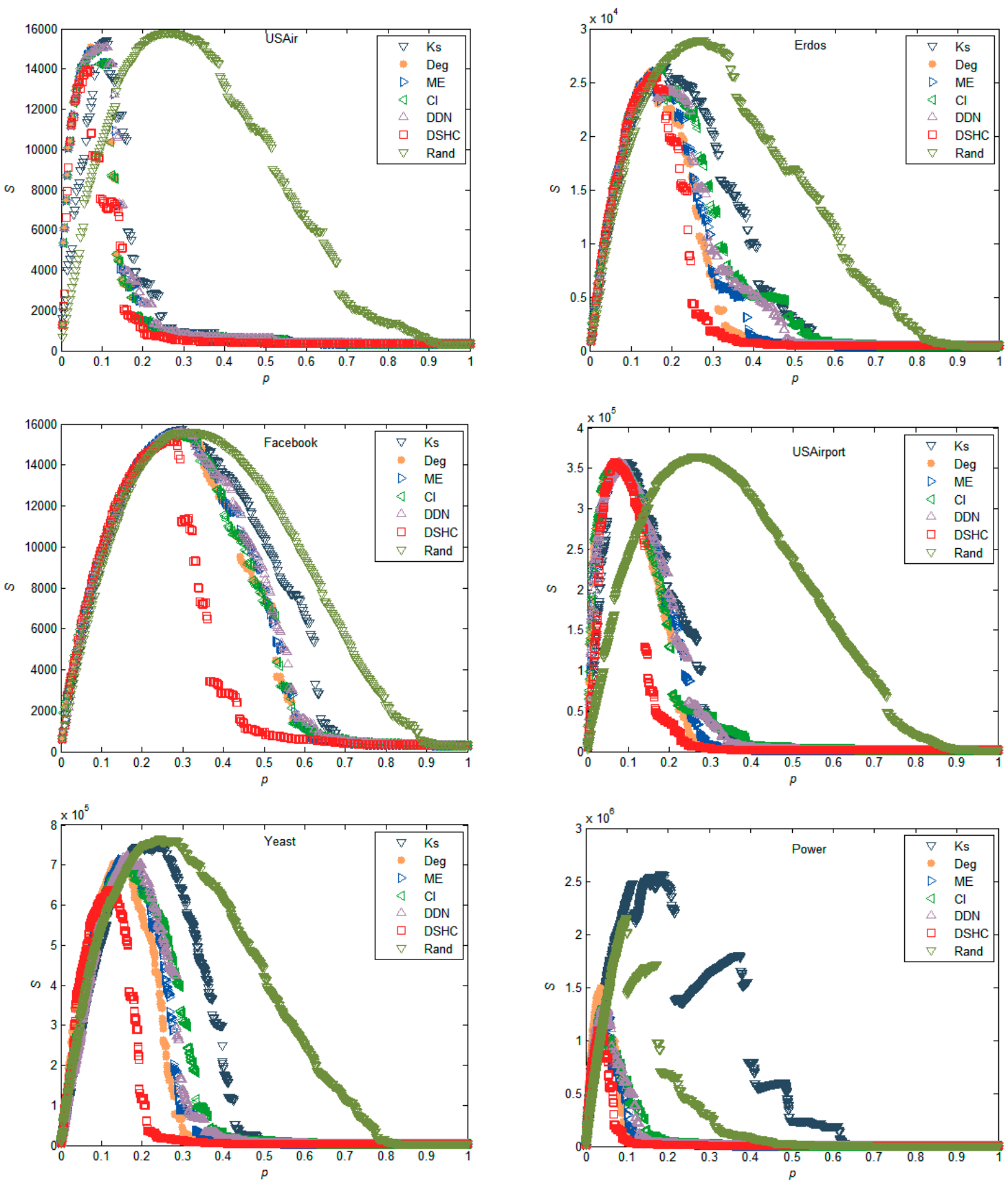

Figure 3. The network susceptibility (S) subjects with different attack strategies.

We further compare the changes in the decline rate of network efficiency when nodes are removed based on different importance sequences calculated by k-shell, degree, ME, CI, DDN, DSHC, and Rand. It can be observed that nodes removal according to the proposed method results in the largest decrease of the network efficiency. Taking network Facebook network in Figure 4 as an example, when $p$ changes from 0.1 to 0.5 , the curve corresponding to the DSHC algorithm is always higher than the curve represented by other algorithms. In other networks, it can also be seen that DSHC algorithm can achieve better results in a larger scale of $p$. 

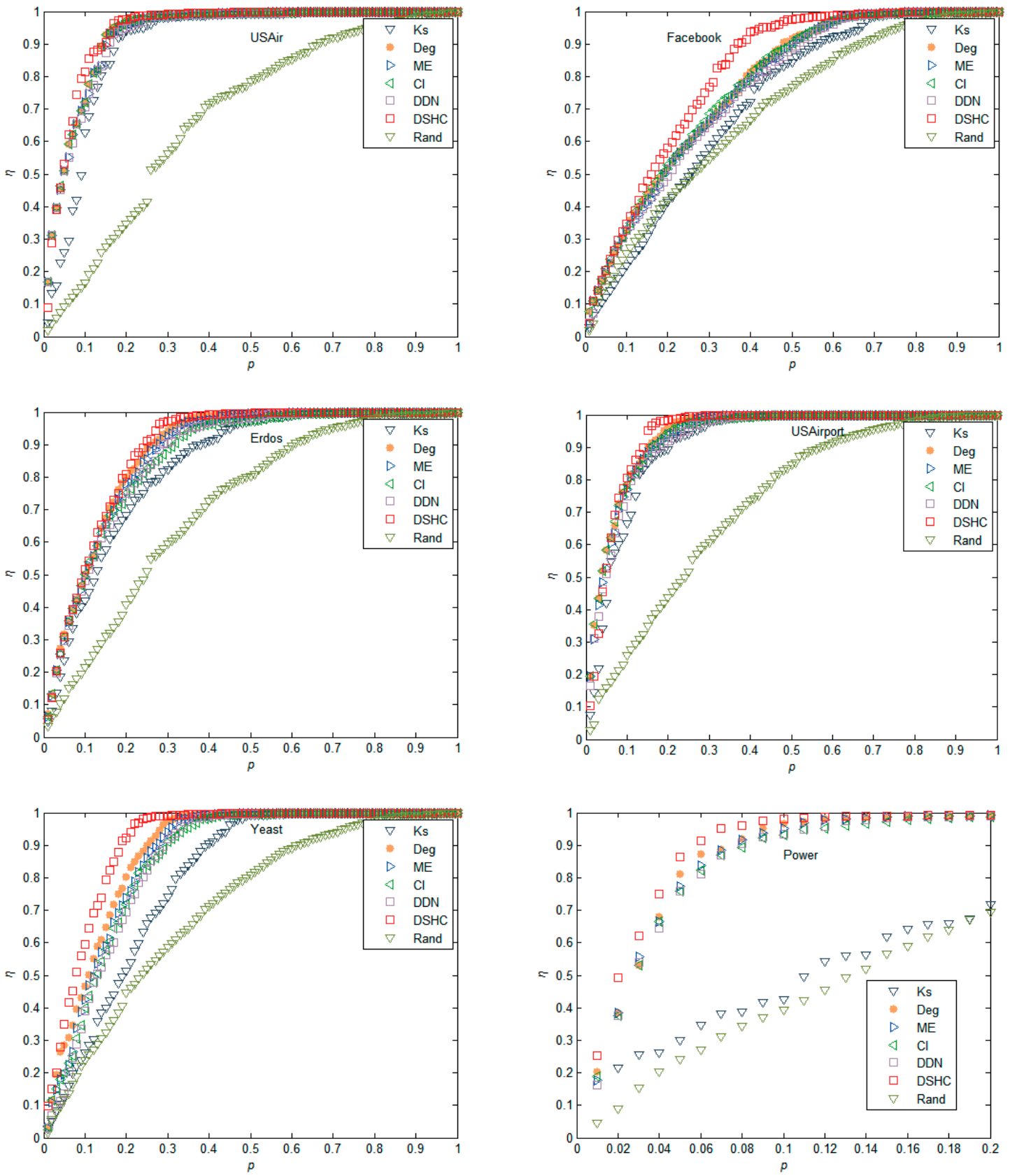

Figure 4. The decline rate of network efficiency $(\eta)$ as a monotonic function of the number of blocked nodes as compared with k-shell, degree, ME, collective influence algorithm (CI), DDN, and Rand in USAir, Erdos, Facebook, USAirport, Yeast, and Power networks, respectively.

\subsection{Experiments in Synthetic Networks}

In addition to the real network, we also verify our approach on synthetic small-world networks. We generate three small-world networks with $N=1000$ by a configuration model [47], the number of neighbor nodes of each node in the nearest-neighbor coupled network is $\alpha=8$. We adjust the density of the network by adjusting the randomization reconnection probabilities $\mu$, and generate three network datasets with $\mu=0.06,0.08,0.1$, respectively. From the results in Figure 5, we find that our approach generally outperforms the five existing methods. This is especially true when the networks have a low level of clustering, as shown in Figure 5b,c. This is because, in a network with a low level of clustering, the structural hole characteristics of the nodes in the network are more obvious, and therefore our algorithm produces an advantage. For a highly clustered network, removing nodes whose neighbors 
are densely interconnected with each other hardly blocks communication between nodes' neighbors, because alternative communication channels for its neighbors still exist. Similar results can also be found in Figures 6 and 7. These results imply that nodes with higher structural holes count, and a larger degree are crucial for maintaining the network connectivity.

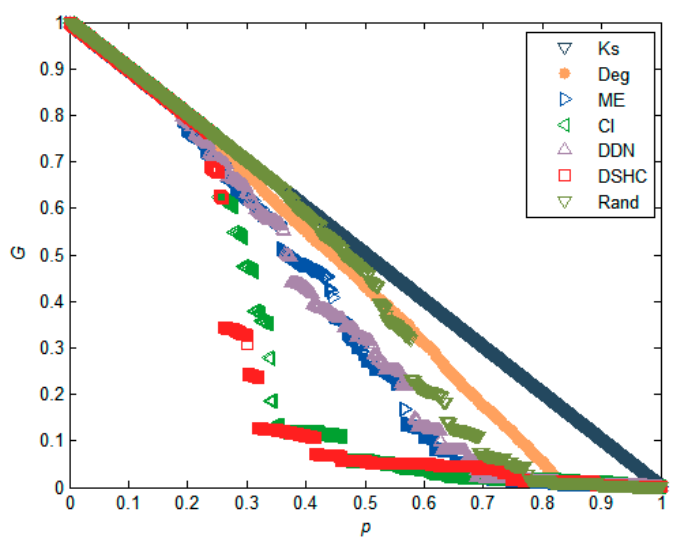

(a)

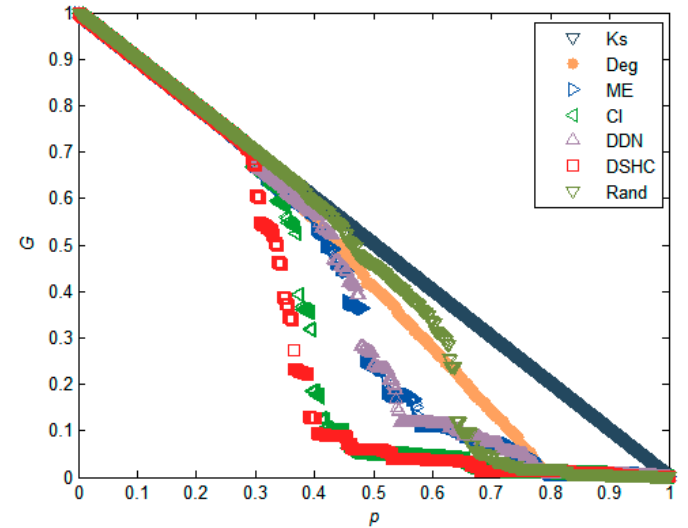

(b)

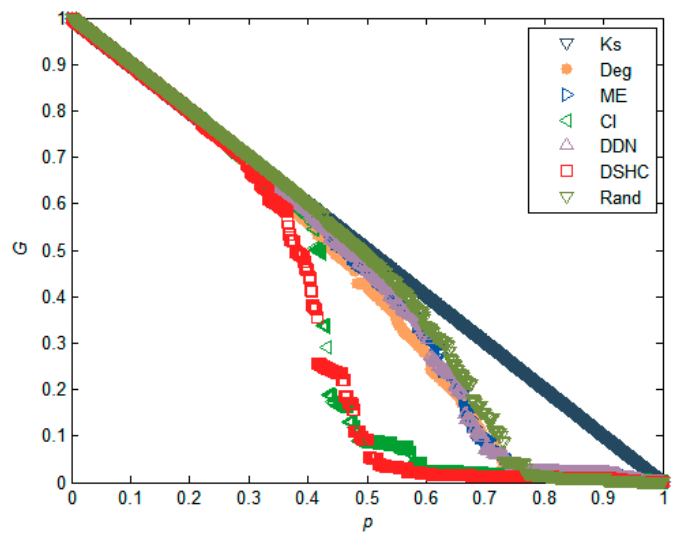

(c)

Figure 5. Comparison of the maximum connectivity coefficient $(G)$ under different attack strategies in synthetic small-world networks, where the size of networks $N=1000$, the number of neighbor nodes of each node in the nearest-neighbor coupled network is $\alpha=8$, and the randomization reconnection probabilities $\mu=0.06,0.08$, and 0.1 for $(\mathbf{a}),(\mathbf{b})$, (c), respectively.

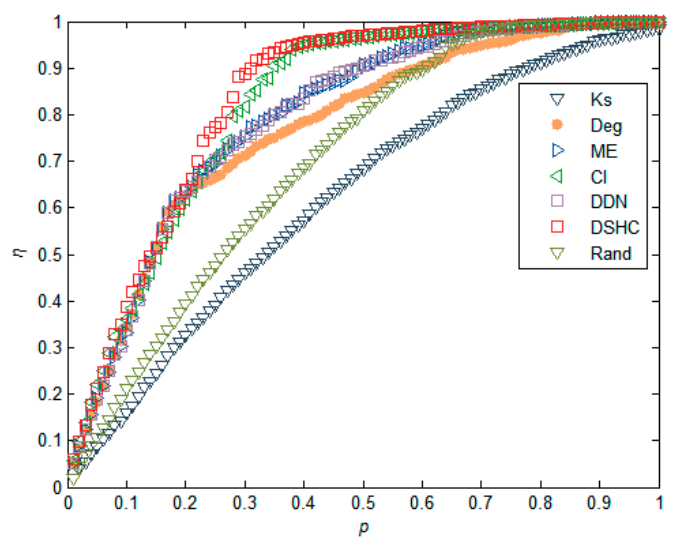

(a)

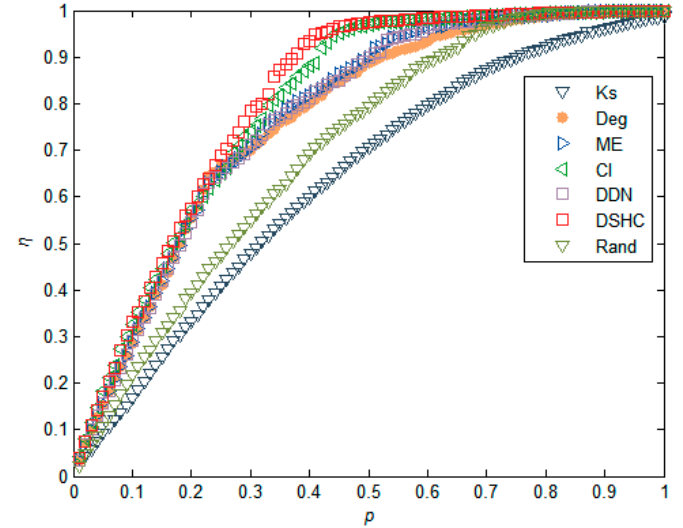

(b)

Figure 6. Cont. 


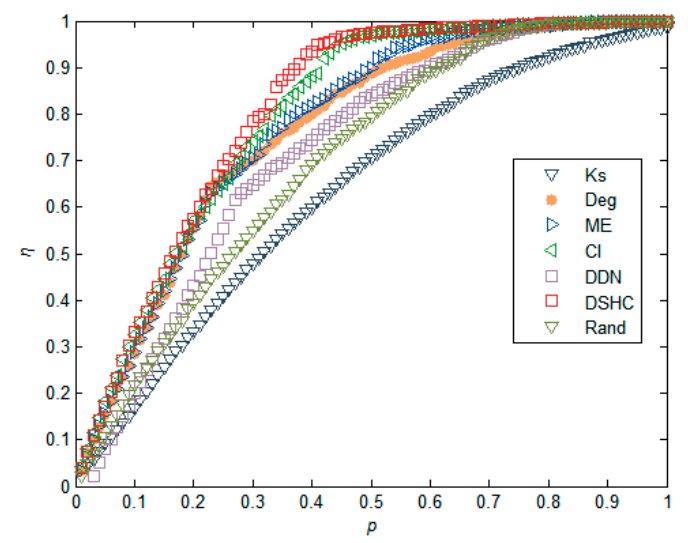

(c)

Figure 6. Comparison of the decline rate of network efficiency $(\eta)$ under different attack strategies in synthetic small-world networks, where the size of networks $N=1000$, the number of neighbor nodes of each node in the nearest-neighbor coupled network is $\alpha=8$, and the randomization reconnection probabilities $\mu=0.06,0.08$, and 0.1 for $(\mathbf{a}),(\mathbf{b}),(\mathbf{c})$, respectively.

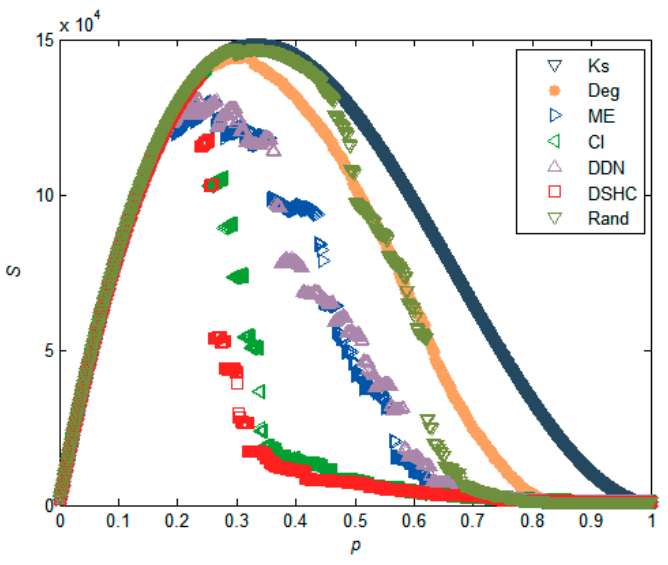

(a)

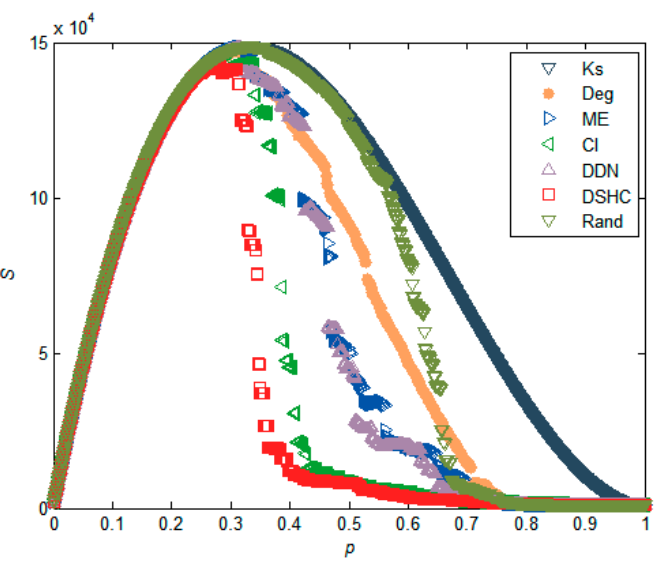

(b)

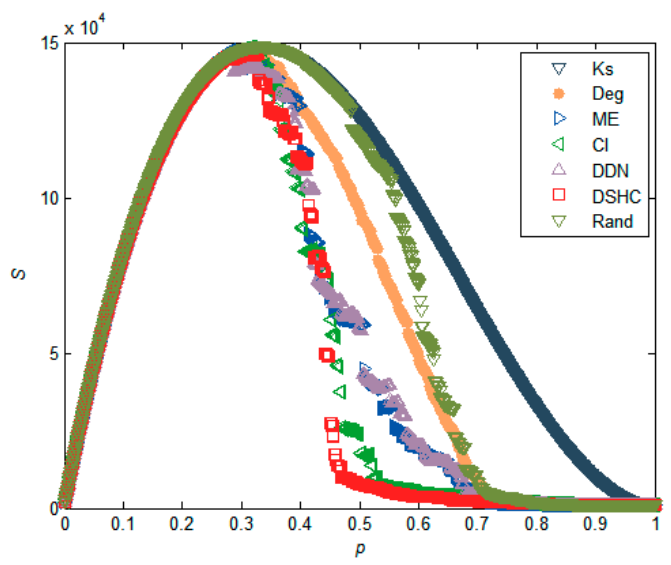

(c)

Figure 7. Comparison of the susceptibility $(S)$ under different attack strategies in synthetic small-world networks, where the size of networks $N=1000$, the number of neighbor nodes of each node in the nearest-neighbor coupled network is $\alpha=8$, and the randomization reconnection probabilities $\mu=0.06$, 0.08 , and 0.1 for $(\mathbf{a}),(\mathbf{b}),(\mathbf{c})$, respectively. 


\section{Conclusions}

Ranking the importance of nodes in complex networks is a challenge of both theoretical and practical significance. In this paper, we leverage the property of structural holes to design a local algorithm to evaluate node importance in maintaining network connectivity. To compare the performance of the proposed measurement with other ranking methods, we investigate the node importance for network connectivity on real and synthetic networks. The experimental results show that our method can outperform the k-shell, degree, ME, CI, DDN, and random sort algorithm. In addition to this, comparisons with the methods using global information, show that the method based on the topological characteristics depends merely on local connectivity patterns, which is much more suitable for large-scale networks.

The algorithm proposed in this paper is used mainly in undirected and unweighted networks. When the local information of nodes is obtained, the structural importance of nodes in the network can be calculated whereas, in the real world, there is a cost limit to launching attacks in the network. Therefore, in future work, we should consider the cost of disintegrating nodes on the design of the node importance ranking algorithm.

Author Contributions: Conceptualization, H.Y.; methodology S.A.; writing-original draft preparation H.Y.; writing-review and editing, H.Y. and S.A. All authors have read and agreed to the published version of the manuscript.

Funding: This research received no external funding.

Conflicts of Interest: The authors declare no conflict of interest.

\section{References}

1. Lü, L.Y.; Chen, D.B.; Ren, X.L.; Zhang, Q.M.; Zhou, T. Vital nodes identification in complex networks. Phys. Rep. 2016, 650, 1-63. [CrossRef]

2. Albert, R.; Barabási, A.L. Statistical mechanics of complex networks. Rev. Mod. Phys. 2002, 74, 47-97. [CrossRef]

3. Amini, M.H.; Arasteh, H.; Siano, P. Sustainable smart cities through the lens of complex interdependent infrastructures: Panorama and state-of-the-art. In Sustainable Interdependent Networks II; Springer: Cham, Switzerland, 2019; pp. 45-68.

4. Liu, J.G.; Ren, Z.M.; Guo, Q.; Wang, B.H. Node importance ranking of complex networks. Acta Phys. Sin. 2013, 62, 178901.

5. Wang, X.F.; Li, X.; Chen, G.R. Complex Networks Theory and Its Application; Tsinghua University Press: Beijing, China, 2006; pp. 1-100.

6. Yu, C.H.; Doppler, K.; Ribeiro, C.B.; Tirkkonen, O. Resource sharing optimization for device-to-device communication underlaying cellular networks. IEEE Trans. Wirel. Commun. 2011, 10, 2752-2763.

7. Liu, H.K.; Zhou, T. Empirical study of Chinese city airline network. Acta Phys. Sin. 2007, 56, 106-111.

8. Zhao, Y.; Du, W.; Chen, S. Application of complex network theory to Urban transportation network analysis. Urban Transp. China 2009, 7, 57-65.

9. Cai, K.Q.; Zhang, J.; Du, W.B.; Cao, X.B. Analysis of the Chinese air route network as a complex network. Chin. Phys. B 2012, 21, 028903. [CrossRef]

10. Watts, D.J;; Dodds, P.S.; Newman, M.E.J. Identity and search in social networks. Science 2012, 296, 1302-1305. [CrossRef]

11. Fang, X.; Misra, S.; Xue, G.; Yang, D. Smart grid-The new and improved power grid: A survey. IEEE Commun. Surv. Tutor. 2012, 14, 944-980. [CrossRef]

12. Callaway, D.S.; Newman, M.E.J.; Strogatz, S.H.; Watts, D.J. Network Robustness and Fragility: Percolation on Random Graphs. Phys. Rev. Lett. 2000, 85, 5468. [CrossRef]

13. Cohen, R.; Erez, K.; ben-Avraham, D.; Havlin, S. Breakdown of the Internet under Intentional Attack. Phys. Rev. Lett. 2001, 86, 3682. [CrossRef]

14. Hao, Y.H.; Han, J.H.; Lin, Y.; Liu, L. Vulnerability of complex networks under three-level-tree attacks. Physica A 2016, 462, 674-683. [CrossRef] 
15. Ruan, Y.R.; Lao, S.Y.; Wang, J.D.; Bai, L.; Chen, L.D. Node importance measurement based on neighborhood similarity in complex network. Acta Phys. Sin. 2017, 66, 38902.

16. Ruan, Y.R.; Lao, S.Y.; Xiao, Y.D.; Wang, J.D.; Bai, L. Identifying influence of nodes in complex networks with coreness centrality: Decreasing the impact of densely local connection. Chin. Phys. Lett. 2016, 33, 028901.

17. Albert, R.; Jeong, H.; Barabási, A.L. Internet: Diameter of the world-wide web. Nature 1999, 401, $130-131$. [CrossRef]

18. Nie, T.Y.; Guo, Z.; Zhao, K.; Lu, Z.M. Using mapping entropy to identify node centrality in complex networks. Physica A 2016, 453, 290-297. [CrossRef]

19. Morone, F.; Makse, H.A. Influence maximization in complex networks through optimal percolation. Nature 2015, 524, 65-68. [CrossRef]

20. Chen, D.B.; Lü, L.Y.; Shang, M.S.; Zhang, Y.C.; Zhou, T. Identifying influential nodes in complex networks. Physica A 2012, 391, 1777-1787. [CrossRef]

21. Bae, J.; Kim, S. Identifying and ranking influential spreaders in complex networks by neighborhood coreness. Physica A 2014, 395, 549-559. [CrossRef]

22. Lü, L.Y.; Zhou, T.; Zhang, Q.-M.; Stanley, H.E. The h-index of a network node and its relation to degree and coreness. Nat. Commun. 2016, 7, 10168. [CrossRef]

23. Hage, P.; Harary, F. Eccentricity and centrality in networks. Soc. Netw. 1995, 17, 57-63. [CrossRef]

24. Stephenson, K.; Zelen, M. Rethinking centrality: Methods and examples. Soc. Netw. 1989, 11, 1-37. [CrossRef]

25. Katz, L. A new status index derived from sociometric analysis. Psychometrika 1953, 18, 39-43. [CrossRef]

26. Sabidussi, G. The centrality index of a graph. Psychometrika 1996, 31, 581-603. [CrossRef] [PubMed]

27. Estrada, E.; Rodriguez-Velazquez, J.A. Subgraph centrality in complex networks. Phys. Rev. E 2005, 71, 056103. [CrossRef]

28. Brin, S.; Page, L. The anatomy of a large-scale hypertextual web search engine. Web Conf. 1998, 30, 107-117. [CrossRef]

29. Kleinberg, J.M. Authoritative sources in a hyperlinked environment. J. ACM 1999, 46, 604-632. [CrossRef]

30. Bonacich, P. Factoring and weighting approaches to status scores and clique identification. J. Math. Sociol. 1972, 2, 113-120. [CrossRef]

31. Lü, L.Y.; Zhang, Y.C.; Yeung, C.H.; Zhou, T. Leaders in social networks, the delicious case. PLoS ONE 2011, 6, e21202. [CrossRef]

32. Weng, J.; Lim, E.P.; Jiang, J.; He, Q. Twitterrank: Finding topic-sensitive influential twitterers. In Third ACM International Conference on Web Search and Data Mining; ACM Press: New York, NY, USA, 2010; pp. 261-270.

33. Burt, R.S. Structural Holes: The Social Structure of Competition; Harvard University Press: Cambridge, MA, USA, 2009.

34. Wang, J.W.; Rong, L.L.; Guo, T.Z. A new measure method of network node importance based on local characteristics. J. Dalian Univ. Technol. 2010, 50, 822-826.

35. Kitsak, M.; Gallos, L.K.; Havlin, S.; Liljeros, F.; Muchnik, L.; Eugene Stanley, H.; Makse, H.A. Identification of influential spreaders in complex networks. Nat. Phys. 2010, 6, 888-893. [CrossRef]

36. Cheng, X.Q.; Ren, F.X.; Shen, H.W.; Zhang, Z.K.; Zhou, T. Bridgeness: A local index on edge significance in maintaining global connectivity. J. Stat. Mech. Theory Exp. 2010, 10, 10011. [CrossRef]

37. Dereich, S.; Mörters, P. Random networks with sublinear preferential attachment: The giant component. Ann. Probab. 2013, 41, 329-384. [CrossRef]

38. Vragovi'c, I.; Louis, E.; Díaz-Guilera, A. Efficiency of informational transfer in regular and complex networks. Phys. Rev. E 2005, 71, 36122. [CrossRef]

39. Latora, V.; Marchiori, M. A measure of centrality based on network efficiency. New J. Phys. 2007, 9, 188. [CrossRef]

40. Blagus, N.; Šubelj, L.; Bajec, M. Self-similar scaling of density in complex real-world networks. Physica A 2012, 391, 2794-2802. [CrossRef]

41. Pajek Datasets. Available online: http://vlado.fmf.unilj.si/pub/networks/pajek/data/gphs.htm (accessed on 1 January 2006).

42. Batagelj, V.; Mrvar, A. Pajek-program for large network analysis. Connections 1998, 21, 47-57.

43. Zeng, A.; Liu, W. Enhancing network robustness against malicious attacks. Phys. Rev. E 2012, 85, 66130. [CrossRef] 
44. Sun, X.; Gollnick, V.; Wandelt, S. Robustness analysis metrics for worldwide airport network: A comprehensive study. Chin. J. Aeronaut. 2017, 30, 500-512. [CrossRef]

45. Von Mering, C.; Krause, R.; Snel, B.; Cornell, M.; Oliver, S.G.; Fields, S.; Bork, P. Comparative assessment of large-scale data sets of protein-protein interactions. Nature 2002, 417, 399-403. [CrossRef]

46. Guelzim, N.; Bottani, S.; Bourgine, P.; Kepes, F. Topological and causal structure of the yeast transcriptional regulatory network. Nat. Genet. 2002, 31, 60-63. [CrossRef]

47. Watts, D.J.; Strogatz, S.H. Collective dynamics of small-world networks. Nature 1998, 393, 440-442. [CrossRef] [PubMed]

(C) 2020 by the authors. Licensee MDPI, Basel, Switzerland. This article is an open access article distributed under the terms and conditions of the Creative Commons Attribution (CC BY) license (http://creativecommons.org/licenses/by/4.0/). 